\title{
MAGNETIC FIELD MEASUREMENT OF THE AIR SLOT DIPOLE MAGNET
}

\author{
M. Umezawa*, K. Hiramoto, Power \& Industrial Systems R \& D Laboratory, Hitachi Ltd., \\ 7-2-1, Omika-cho, Hitachi-shi, Ibaraki-ken, 319-1221, Japan, \\ M. Tadokoro, J. Hirota, Hitachi Works, Hitachi Ltd., \\ 3-1-1, Saiwai-cho, Hitachi-shi, Ibaraki-ken, 317-8511, Japan
}

\begin{abstract}
Field measurement results of the air slot type dipole magnet are presented. The vacant portion, called the air slot, located in the iron core controls the flow of the magnetic flux and improves uniformity of the magnetic field. This magnet realizes a better magnetic field distribution over a wide range of field strengths. The prototype of the magnet had been fabricated and tested to verify the design principle. The results of measurements by the Hall-probe showed good agreement with the numerical calculations for magnetic field strengths ranging from $0.27 \mathrm{~T}$ to $1.83 \mathrm{~T}$. The field uniformity $\Delta \mathrm{B} / \mathrm{B}$ was below $0.04 \%$ in the good field region.
\end{abstract}

\section{INTRODUCTION}

We have proposed a new design concept for bending magnets which can improve magnetic field distribution[1]. The design concept is based on control of the flow of the magnetic flux in the magnet pole by introducing vacant portions into the magnet iron core. This new concept was proposed originally to develop a combined function bending magnet[2]. Effectiveness had been confirmed by numerical study[3] and the results of further study will be presented[4].

In the conventional dipole bending magnet, application of shims to the magnet pole face has been considered an effective way of improving magnetic field uniformity. However, the shape of the shims for very high fields often had a bad influence on the field distribution at low magnetic field, and their shape for low field is not suitable for high magnetic field. In order to obtain a good field distribution over a wide range of magnetic field strengths, it is necessary to overcome this problem. Therefore, we applied the present design concept to dipole bending magnets and confirmed by numerical study that the vacant portion in the iron core can improve the magnetic field distribution at various excitation levels. Additionally, the air slot type pole width can be narrower than that of the conventional type and the yoke size and weight of the magnet can be smaller. Because of these advantages, the air slot type magnet is widely applicable as a synchrotron magnet and for beam transport, rotating gantry, and so on.

\footnotetext{
”Email: umezawa@erl.hitachi.co.jp
}

The dipole magnet for a compact proton synchrotron[5], which has two air slots in its magnet pole, has been investigated by two- and three- dimensional numerical calculations[1]. In order to verify this new design principle, that is the effect of the air slots, the prototype of this magnet was constructed and magnetic field measurements were made with the Hall-probe for magnetic field strengths from $0.27 \mathrm{~T}$ to $1.82 \mathrm{~T}$. The measurement results showed that the air slots had their designed effect.

\section{DESIGN OF THE MAGNET}

\subsection{Design Principle}

The scheme for improving the magnetic field distribution by introducing air slots in the magnet pole is outlined below. The magnetic susceptibility of the air slots is much lower than that of the iron core. At high magnetic field, the perimeter of the iron pole saturates earlier than the central area as excitation goes up. On the other hand, introducing air slots in the central area leads to an increase of the magnetic flux density in the iron region around the air slots. If the position and shape of the air slots are adjusted to equalize the magnetic flux density in the whole pole iron at high field, the magnetic field distribution can be flattened. At low magnetic field where the magnetic flux does not saturate, the effect of air slots is very small and the magnetic field distribution is controlled only by the shape of the pole surface. This new concept is useful for improving the dynamic range of the magnetic field and extending the good field region size, if both slotting and shimming would be used together.

\subsection{Design Requirements}

We designed the air slot type bending magnet for a compact proton synchrotron[5]. In this synchrotron, the beam can be accelerated from $7 \mathrm{MeV}$ up to $270 \mathrm{MeV}$ at a repetition cycle of $0.5 \mathrm{~Hz}$ for proton radiography as well as cancer treatment. The magnet yoke is made of stamped laminated steel of $0.5 \mathrm{~mm}$ thickness to reduce the eddy current effect. The mean radius and the circumference of the synchrotron are about $3.5 \mathrm{~m}$ and $23 \mathrm{~m}$, respectively, so the curvature radius of the bending magnet is $1.4 \mathrm{~m}$. In order to shorten the straight section for the extraction deflector, the deflection by the $22^{\circ}$ defocusing edge angle 
of the bending magnet is needed. The minimum magnetic field strength is $0.273 \mathrm{~T}$ for beam injection and the maximum is $1.808 \mathrm{~T}$ for extraction. The good field region is determined based on our analysis of the beam size in the synchrotron. The specifications of the magnet are listed in Table 1.

Table 1: Specifications of the dipole magnet

\begin{tabular}{l|l}
\hline Minimum Magnetic Field (T) & 0.273 \\
Maximum Magnetic Field (T) & 1.808 \\
Curvature Radius (m) & 1.4 \\
Bending Angle (degree) & 60 \\
Field Length (m) & 1.466 \\
Edge Angle (degree) & 22 \\
Gap Height (mm) & 56 \\
Good Field Region (mm) & \pm 50 \\
Required Magnetic Field Uniformity (\%) & 0.04 \\
\hline
\end{tabular}

\subsection{Calculation Results}

Based on these specifications, the magnet shape is determined using the computer code PANDIRA. The air slot type magnet design is shown in Fig. 1. The pole width is $300 \mathrm{~mm}$ and quarter circles are used to prevent the local edge effect at both ends of the pole. This pole width can be narrower than that of the shimmed type magnet[1] due to the effect of the air slot. Air slots have an oval shape with $70 \mathrm{~mm}$ length and $10 \mathrm{~mm}$ width. Figure 2 shows two-dimensional magnetic field distribution. The horizontal and vertical axes show, the radial distance from the design orbit and the deviation of the magnetic field strength from the value on the design orbit $(r=0)$, respectively. The packing factor is assumed to be 0.98 .

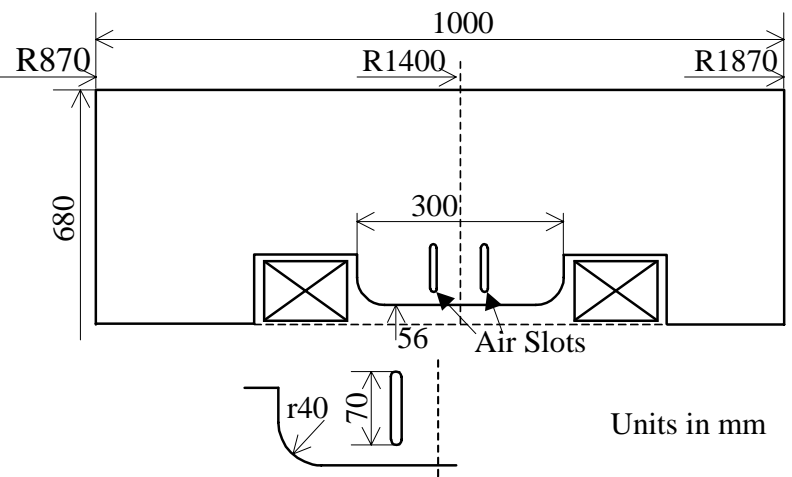

Figure 1: Cross-sectional view of the air slot type magnet

As shown in Fig. 2, the good field region is wider than $\pm 60 \mathrm{~mm}$, and the magnetic field distribution does not change very much from $0.27 \mathrm{~T}$ to $1.81 \mathrm{~T}$. The position, length and width of the air slot are adjusted to flatten the magnetic field distribution at various field strengths.

In order to decide the magnet end shape and estimate the effective edge angle and multipole components of the magnetic field, three-dimensional field calculations were performed using the computer code TOSCA. Both ends of the magnet are designed to approximate Rogowski's curve to avoid the saturation at the sharp edge of iron core by 4 step cuts in a $60 \mathrm{~mm}$ long region along the orbit.

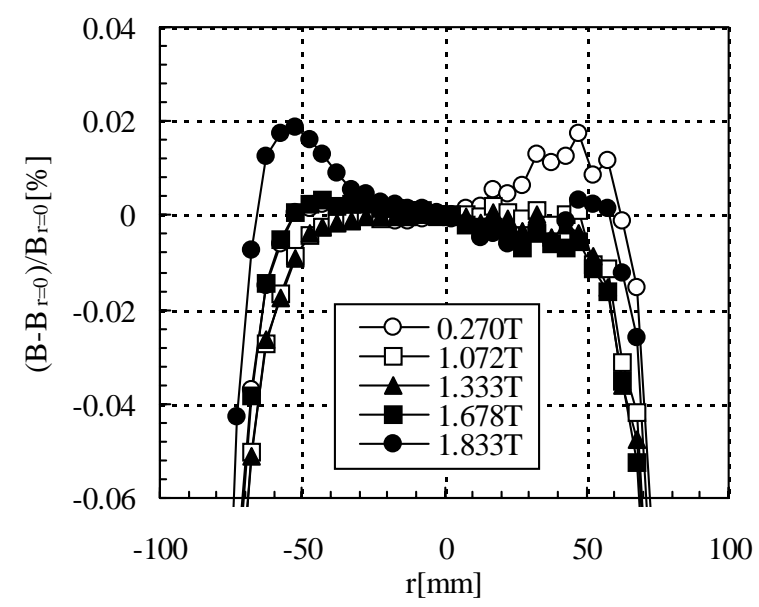

Figure 2: Two-dimensional magnetic field distributions of the air slot type magnet

\section{FIELD MEASUREMENT AND ANALYSIS}

Figure 3 shows the prototype dipole magnet which was fabricated to verify the design as mentioned above. The magnetic field measurements were made using the Hallprobe mounted on the arm of a computer-controlled positioning $x-y-z$ table. The absolute calibration of the system was established by comparison with NMR probe measurements. The interval of the mesh point for the midplane measurements was less than or equal to $10 \mathrm{~mm}$ inside the gap and $500 \mathrm{~mm}$ outside it in beam direction. Analyses of measurement results at various excitations were performed in the same way as for the calculation results.

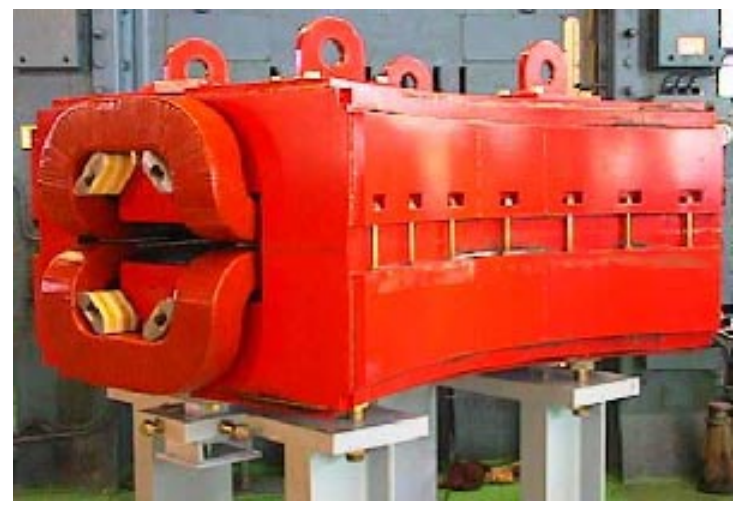

Figure 3: The prototype dipole magnet

Results of the excitation are compared with the results calculated by PANDIRA in Fig. 4. The vertical axis shows the magnetic field strength at the center of the magnet. The excitation current measured at $1.83 \mathrm{~T}$ is $10 \%$ 
larger than that of the linear case because of saturation of the iron core.

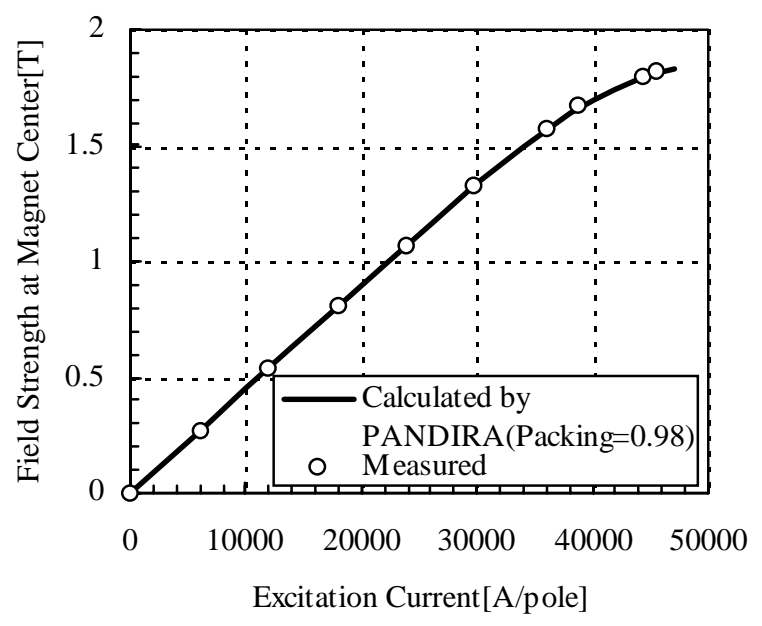

Figure 4: Magnetic field strength as a function of excitation current

Figure 5 shows the measured uniformity of the magnetic field at various excitations. Deviations of the field strength are less than $0.04 \%$ in the good field region at all excitation levels. Comparing results of the calculation (Fig. 2) and measurements (Fig. 5) shows some deviations, about $0.01 \%$ order, which can be expected from uncertainties in the manufacturing process. These results show that the effect of the air slots performs as designed.

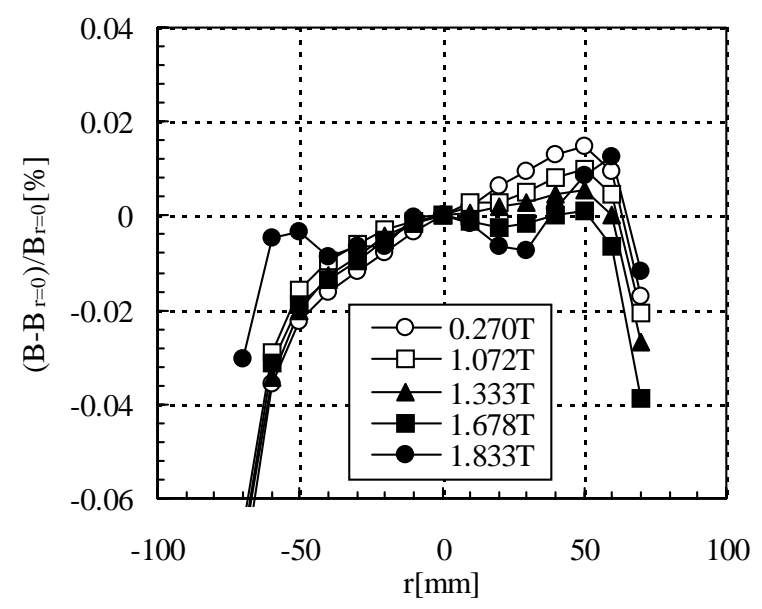

Figure 5: Measured uniformity of magnetic field strength

To estimate the effect of the non-linear components, the field strength as a function of distance in the radial direction is fitted by a Taylor expansion and the components are integrated along the beam reference orbit. The sextupole component normalized by magnetic rigidity is $-0.03 \mathrm{~m}^{-2}$ at injection and over $-0.2 \mathrm{~m}^{-2}$ at field strength $1.83 \mathrm{~T}$ because of the saturation effect at magnet end. These values can be expected based on threedimensional calculations. We confirmed by particle tracking calculations that the operation of the synchrotron from injection to extraction can be done on the assumption that the multipole components mentioned above are included in the magnetic field at fringing region.

\section{CONCLUSION}

We carried out magnetic field measurements of the air slot type magnet to verify our design principle. The air slots in the magnet pole improve the magnetic field distribution by controlling the flow of magnetic flux. Using the air slot type design, we can obtain the magnet which has a good field distribution at several excitation levels. The size and weight can be reduced because of the narrower pole width achieved by the effect of the air slots. Because of these advantages, this magnet design can be applied widely to synchrotron, beam transport, rotating gantry, and so on.

The prototype of the magnet was constructed for the main dipole of a compact proton synchrotron. Measurements using the Hall-probe were consistent with two- and three- dimensional calculation results. The results verified that the design principle which we proposed was valid and the air slots in the iron core performed as desired. Detailed analysis of the measurement results showed that the model magnet will achieve the specifications of the compact synchrotron we designed.

\section{REFERENCES}

[1] M. Umezawa et al., "A New Dipole Bending Magnet with Improved Magnetic Field Distribution", Proc. of the 11th Symposium on Accelerator Science and Technology, Spring-8, Japan(1997)380382.

[2] M. Tadokoro et al., "A Combined Function Magnet for a Compact Synchrotron", Proc.of 1996 Part. Accel. Conf., Vancouver, Canada (1996) 3294-3296.

[3] A. Noda et al., "Development of Compact Proton Synchrotron with Combined Function Dedicated for Cancer Therapy", Proc. of the 11th Symposium on Accelerator Science and Technology, Spring-8, Japan (1997)314-316.

[4] A. Noda et al., "A Compact Proton Synchrotron with CombinedFunction Lattice dedicated for Cancer Therapy", Proc. of this conference.

[5] K. Hiramoto et al., "A Compact Synchrotron for Cancer Treatment", Proc. of 1996 Part. Accel. Conf., Vancouver, Canada(1996)3813-3815 